\title{
Structure and Stability of Pristine and Bi and/or Sb Decorated Titanate Nanotubes
}

\author{
Andrea Rónavári ${ }^{1}$, Balázs Buchholcz ${ }^{1}$, Ákos Kukovecz ${ }^{1,2}$, Zoltán Kónya ${ }^{1,3}$ \\ ${ }^{1}$ University of Szeged, Department of Applied and Environmental Chemistry, H-6720 Szeged, \\ Rerrich ter 1, Hungary \\ ${ }^{2}$ MTA-SZTE “Lendület” Porous Nanocomposites Research Group, H-6720 Szeged, Rerrich \\ ter 1, Hungary \\ ${ }^{3}$ MTA-SZTE Reaction Kinetics and Surface Chemistry Research Group, H-6720 Szeged, \\ Rerrich ter 1, Hungary
}

\begin{abstract}
$\underline{\text { Abstract }}$
The 90-100 nm long protonated titanate nanotubes were synthesized by hydrothermal method from anatase at $155^{\circ} \mathrm{C}$. The $\mathrm{Bi}$ and $\mathrm{Sb}$ affected crystallization of the tubular titanate structures during calcination was studied by transmission electronmicroscopy (TEM), X-ray diffraction (XRD) and Raman spectroscopy. It was shown that $\mathrm{Bi}$ and $\mathrm{Sb}$ have different effects on the transformation; while Bi enhanced the crystallization of the tubular structure to rutile, the $\mathrm{Sb}$ protected the trititanate form.
\end{abstract}

\section{Introduction}

The discovery of carbon nanotubes encouraged researchers to widen their knowledge in the synthesis and characterization of the nanotubular materials. In the last decades several studies have been published about the synthesis and characterization of different inorganic nanotubes [1], [2], [3]. Titanium oxide based nanotubes have became a particular research subject for their applications to photocatalytic [4], [5], [6], [7], biomedical [8], energy storing [9] and semiconductor devices [10], to name a few.

The first sodium-trititanate nanotube synthesis was showed by Kasuga et al. [11]. The procedure was the hydrothermal treatment of $\mathrm{TiO}_{2}$ powders in $10 \mathrm{M} \mathrm{NaOH}$ solution and needle-shaped nanotubes were obtained after the reaction.

Titanate nanotubes have been used for heterogeneous catalytic studies as support. The Au decorated titanate nanotubes could be promising catalysts in the low-temperature watergas shift reaction [12]. Platinum nanoparticles can improve this behavior [13] as well. $\mathrm{Pd}(\mathrm{II}) /$ titanate nanotubes have high activity and selectivity to the double-bond migration for example in the isomerization of allylbenzene [14]. The photocatalytic activity of trititanate nanotubes is weaker than that of anatase or other titanium-oxide nanocrystals [20] because of the distorted structure and wider band gap. Thus, several studies were published about the photosensitization of the titanate nanotubes. One possible sensitizer is cadmium sulphide, a versatile semiconductor with tunable $2.4-4.2 \mathrm{eV}$ band gap [15] which can be obtained as layered assembly or as core/shell heterostructure [16]. The photocatalytic activity could be improved by framework doping using iron [17] or nitrogen [18] dopants as well.

Metal ion incorporation into titanate nanotubes will definitely change their properties, however, as a result of the metal ion insertion the structure of the nanotubes can be strongly influenced as well. In the present study, we show the affect of Bi and Sb on the heat treatment induced structural changes of the titanate nanotubes.

\section{Experimental}


All the chemicals were of analytical grade. Sodium hydroxide $(\mathrm{NaOH})$ and hydrochloric acid $(\mathrm{HCl})$ were purchased from Molar Chemicals, while titanium dioxide $\left(\mathrm{TiO}_{2}\right.$, Degussa $\mathrm{P}$ 25 ) bismuth(III) acetate and antimony(III) acetate were purchased from Sigma-Aldrich ${ }^{\circledR}$. The reagents were used without further purification. Deionized water was used in all aqueous solution for both preparations and washings.

\section{Titanate nanotubes synthesis}

The titanate nanotubes (TiONTs) were prepared by an alkaline hydrothermal method detailed elsewhere [19], [20], [21], [22], [23], [24]. In a typical synthesis $50 \mathrm{~g}$ of $\mathrm{TiO}_{2}$ powder and $1 \mathrm{~L}$ of $10 \mathrm{M} \mathrm{NaOH}$ aqueous solution were mixed. When a white suspension appeared, it was transferred into a Teflon-lined stainless steel autoclave (diameter $4 \mathrm{~cm}$, height $14 \mathrm{~cm}$ ) and kept at $155^{\circ} \mathrm{C}$ for 24 hours under continuous rotation (around the short axis of the autoclave, with a speed of $3 \mathrm{rpm}$ ). The as-prepared nanotubes were washed thoroughly with deionized water to neutral $\mathrm{pH}$ then subsequently with $0.1 \mathrm{M} \mathrm{HCl}$ solution to $\mathrm{pH} 2$ and finally with water again to obtain a neutral salt-free product. The nanotubes were dried in air at $60^{\circ} \mathrm{C}$.

\section{Ion exchange and heat treatment}

Bismuth-, antimony- and bismuth-antimony/titanate nanocomposites were prepared by ion exchange technique. The titanate nanotubes were dispersed in deionized water by ultrasonication and then bismuth acetate, antimony acetate and bismuth- and antimony acetate was added to the solution (the overall metal content was $10 \mathrm{wt} \%$ for all samples). After stirring for $24 \mathrm{~h}$ the suspension was filtered and washed with deionized water to remove the remaining acetate ions and finally all the samples were dried at $60{ }^{\circ} \mathrm{C}$ for 24 hours. All of the as-prepared samples (including the non-ion exchanged titanate nanotubes) underwent a thermal annealing process in air atmosphere at elevated temperature $\left(100-900{ }^{\circ} \mathrm{C}\right)$ for 1 hour. Samples were removed after each thermal operation for further characterization.

\section{Characterization}

The morphology of the pristine and modified titanate nanotubes was characterized by transmission electron microscopy (TEM). The TEM observations were performed on a FEI Tecnai $\mathrm{G}^{2} 20 \mathrm{X}$-Twin instrument ( $200 \mathrm{kV}$ accelerating voltage) using copper mounted holey carbon grids.

The crystal structure was characterized by X-ray powder diffraction. The scans were performed with a Rigaku MiniFlex II powder diffractometer using $\mathrm{Cu} \mathrm{K} \alpha$ radiation.

Energy dispersive X-ray spectroscopy (EDX) was used to estimate the elemental analysis of the heat treated pristine and ion exchanged samples. The EDX measurements were carried out on a Hitachi S-4700 electron microscope equipped with a Röntec energy dispersive X-ray spectrometer.

Raman spectra of samples were measured at $532 \mathrm{~nm}$ laser excitation using a Thermo Scientific DXR Raman microscope. Scans were integrated at $4 \mathrm{~cm}^{-1}$ resolution until the desired signal-to-noise ratio of 1000 or better was achieved (max. 2 minutes).

Heat treatment was carried out at $100-900{ }^{\circ} \mathrm{C}$ but structural changes appeared only at $500{ }^{\circ} \mathrm{C}$ and higher temperatures, therefore, only those results (as-synthesized and at 600,750 and $900{ }^{\circ} \mathrm{C}$ calcined samples) will be shown here.

\section{Results and discussion}




\section{Morphological and structural properties of the as-synthesized titanate nanotubes}

The hydrothermally synthesized titanate nanotubes are open-ended tubular structures with 90-100 nm length and 8-10 nm width (Figure 1).

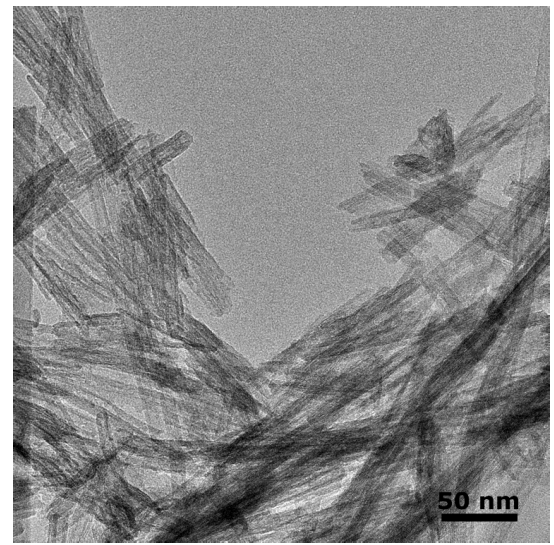

1. Figure TEM image of the as-synthesized titanate nanotubes

Energy dispersive X-ray spectroscopy (EDX) was carried out for the elemental analysis of the different titanate nanotubes (Figure 2). It is obvious that the samples contain $\mathrm{Ti}, \mathrm{O}, \mathrm{Sb}$, $\mathrm{Bi}$ and are free from other elements.

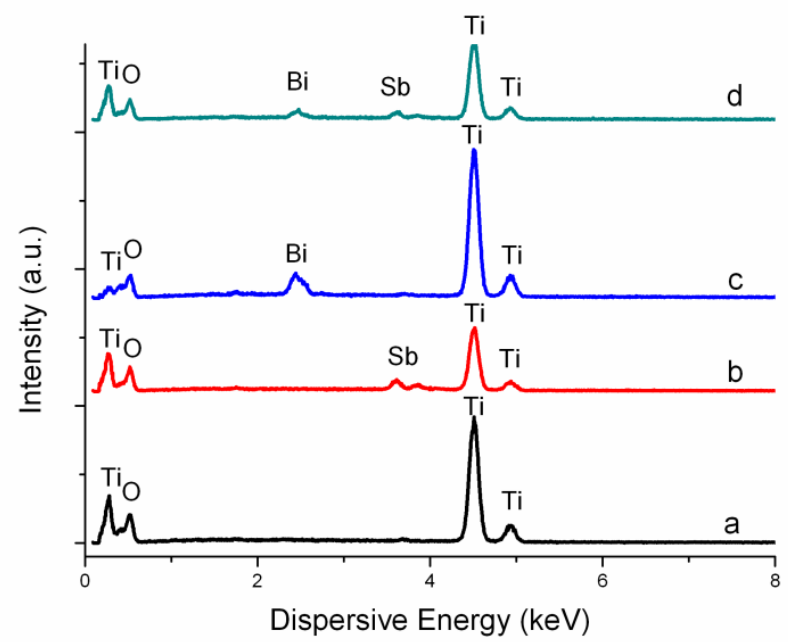

2. Figure EDX spectra of the pristine (a), antimony (b), bismuth (c), and antimony/bismuth (d) modified titanate nanotubes

Figure 3 shows TEM images of pristine, antimony, bismuth, and antimony/bismuth modified titanate nanotubes calcined at $600^{\circ} \mathrm{C}$. It can be clearly seen that the initial tubular structure was somewhat damaged and the nanotubes were transformed into rod-like nanostructures. (Albeit it is not shown here, at $900{ }^{\circ} \mathrm{C}$ the structure of all the samples collapsed entirely and $\mathrm{TiO}_{2}$ nanoparticles were formed.). As it can be seen from Figure 3b, in the presence of $\mathrm{Sb}$ the tubular structure of the titanate nanotubes was protected. On the other hand, the structure of the Bi-containing samples totally collapsed and 10-20 nm sized crystalline nanoparticles were observed.

The picture also shows the presence of homogenously dispersed metal nanoparticles with diameter of less than $5 \mathrm{~nm}$. 

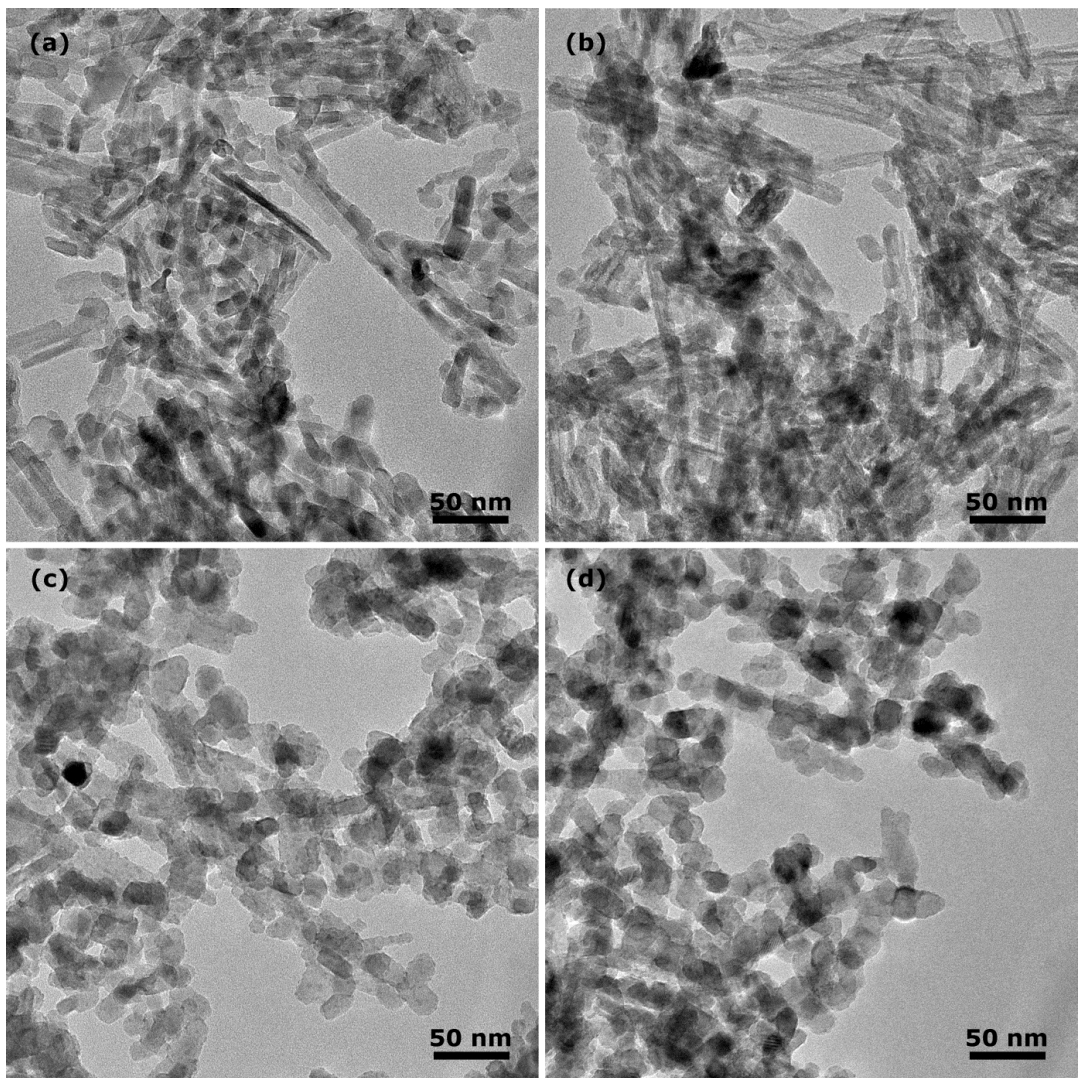

3. Figure TEM images of the pristine (a), antimony (b), bismuth (c), and antimony/bismuth (d) modified titanate nanotubes calcined at $600{ }^{\circ} \mathrm{C}$.

\section{Powder X-ray diffraction study}

The X-ray diffraction pattern (XRD) of the samples revealed the crystalline structure of the nanotubes (Figure 4). The characteristic reflections of the hydrogen titanate nanotubes $\left(\mathrm{H}_{2} \mathrm{Ti}_{3} \mathrm{O}_{7}\right)$ are at $2 \theta=10,24.8,28.2$ and $48.8^{\circ}$ among which the $2 \theta=10^{\circ}$ is the one that belongs to the tubular interlayer distance (Figure 4a).

At elevated temperatures the XRD patterns indicate phase transformation from trititanate via anatase to a mixture composed of predominantly rutile and anatase phases for both samples. However, for the Sb-containing titanate nanotubes the crystallization to anatase is slower than for the the pristine sample calcined at 600 and $750{ }^{\circ} \mathrm{C}$ (Figure $4 \mathbf{b}$ ). On the other hand, the Bi-modified material does not contain any anatase phase even at $900{ }^{\circ} \mathrm{C}$ according to the XRD profiles (Figure 4c).

It is noteworthy that at $900{ }^{\circ} \mathrm{C}$ temperature diffraction pattern of all the appropriate metal oxides $\left(\mathrm{Sb}_{2} \mathrm{O}_{3}, \mathrm{Bi}_{2} \mathrm{O}_{3}\right.$ and $\left.\mathrm{SbBiO}_{4}\right)$ appeared as well. 

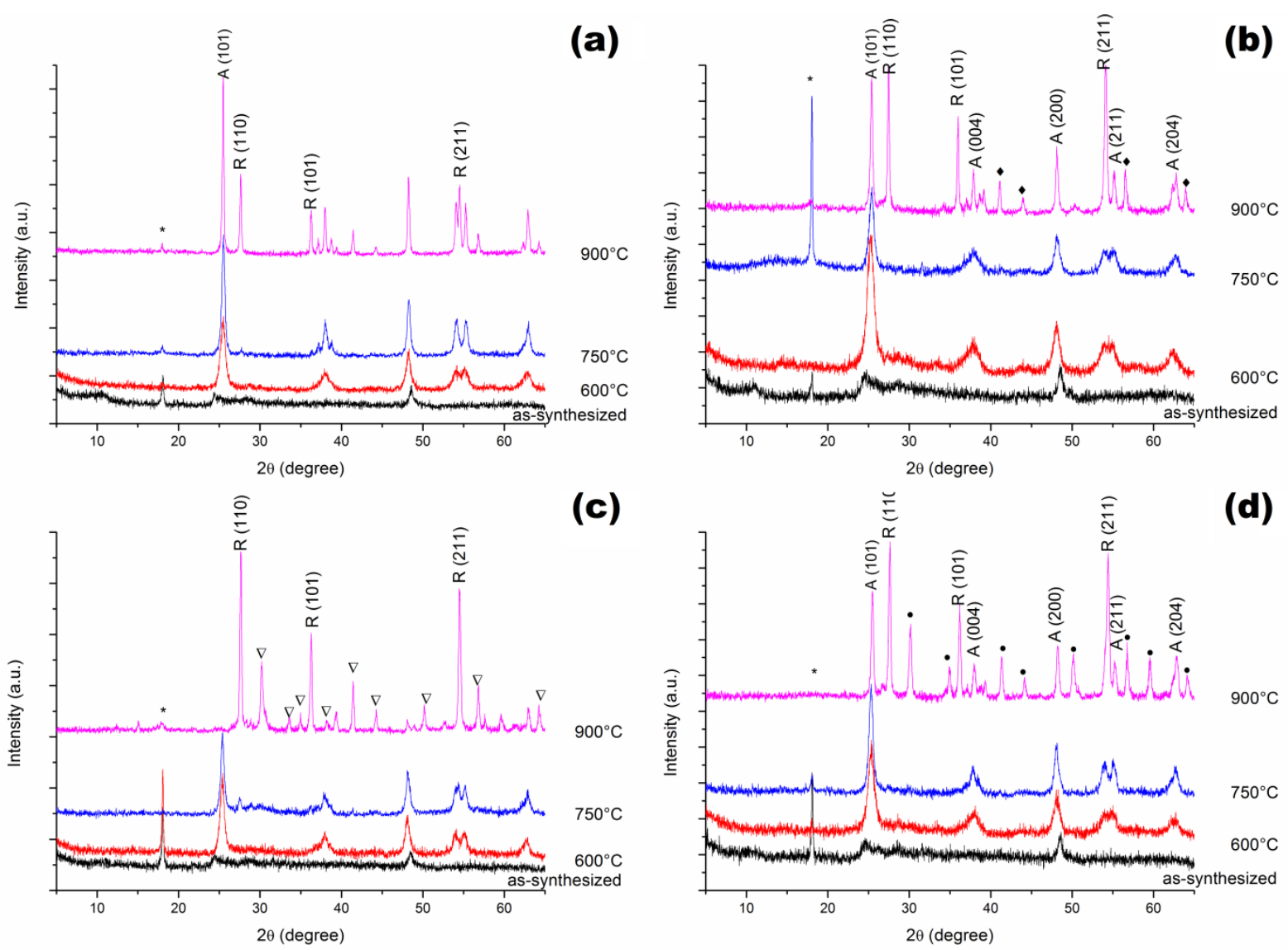

4. Figure X-Ray diffraction profiles of the pristine (a), antimony (b), bismuth (c), and antimony/bismuth (d) modified titanate nanotube samples. The "*" symbolized the reflection of the sample holder, the " $\diamond$ " is the $\mathrm{Sb}_{2} \mathrm{O}_{3}$, the " $\nabla$ " is the $\mathrm{Bi}_{2} \mathrm{O}_{3}$ and the "•" is the $\mathrm{SbBiO}_{4}$.

\section{Raman spectroscopy}

Raman spectra of pristine and heat treated titanate nanotubes are presented in Figure 5. Anatase and rutile have characteristic bands at 142, 194, 394, 512, 635 and 236, 444, 607 $\mathrm{cm}^{-1}$, respectively [25]. Therefore, it can be concluded that the spectrum of the pristine sample Figure 5a does not contain any bands corresponding to an anatase or rutile phase. Our protonated trititanate nanotubes feature characteristic bands at 270,450 and $660 \mathrm{~cm}^{-1}$. The $450 \mathrm{~cm}^{-1}$ band was assigned [26], [27] to a Ti-O-Ti stretching vibration (framework) and the 270 and $660 \mathrm{~cm}^{-1}$ were assigned to Ti-O-M vibrations. In our case $\mathrm{M}$ is $\mathrm{H}^{+}$occupying an ionexchange position in the trititanate wall [28]. The spectrum of the pristine nanotubes and the other spectra as well show these bands which were also reported earlier [26], [27], [28].

The Raman spectra of Sb Figure 5b and the Sb/Bi Figure 5d modified titanate nanotube composites are similar to those of the pristine samples. The Bi containing nanotube spectra Figure 5c. differ from the other ones. The sample which was treated at $900{ }^{\circ} \mathrm{C}$ resembles the spectrum of rutile [25] (see bands at 241, 446 and $610 \mathrm{~cm}^{-1}$ ). 

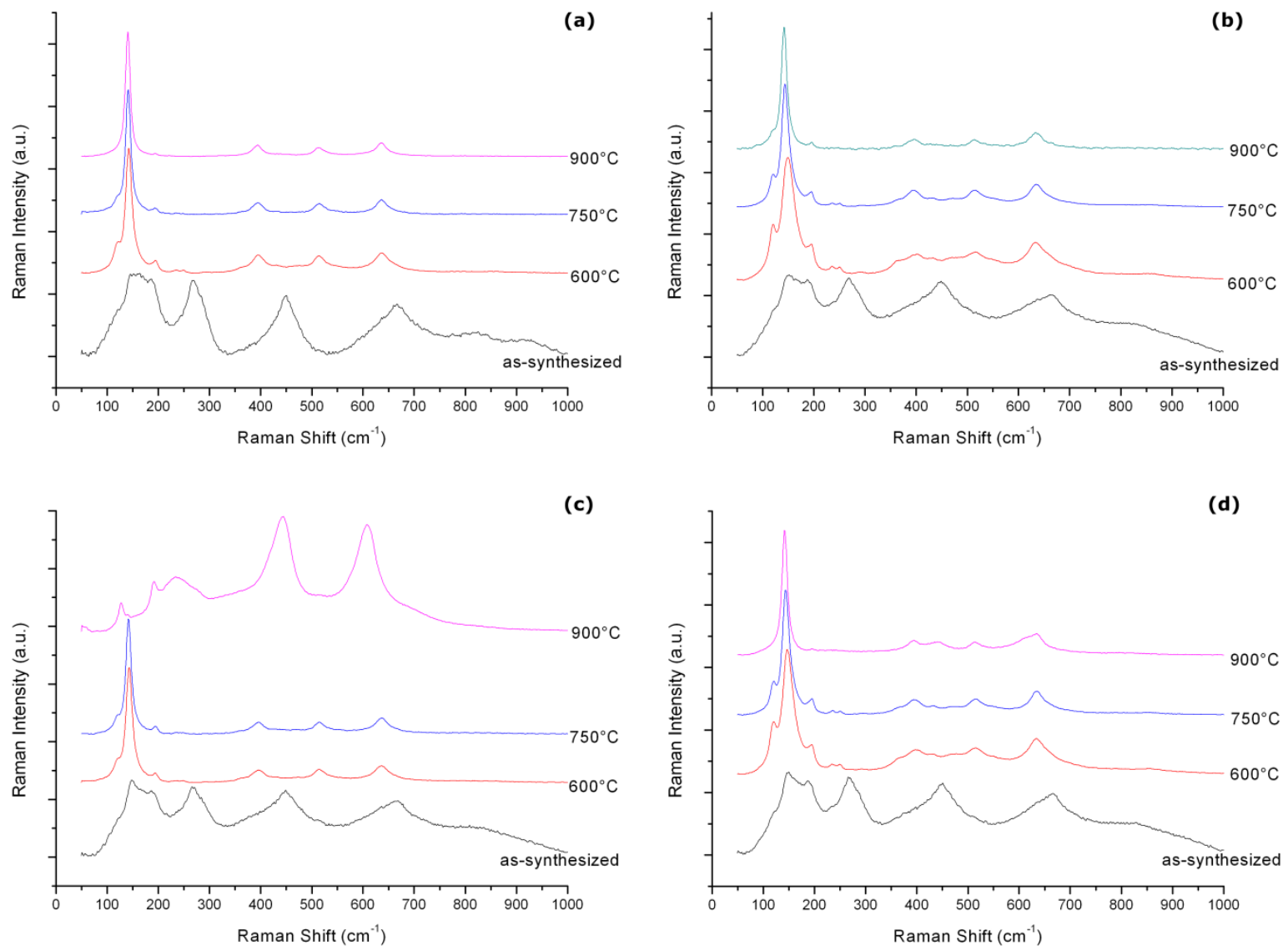

5. Figure Raman spectra of the pristine (a), antimony (b), bismuth (c), and antimony/bismuth (d) modified titanate nanotubes.

\section{Conclusions}

Protonated titanate nanotubes were synthesized by the hydrothermal method at $155{ }^{\circ} \mathrm{C}$ and used for ion exchange experiments. The titanate nanotubes were ion exchanged with antimony, bismuth and antimony/bismuth and then all samples were calcined at different temperatures. The synthesized nanostructures were studied by transmission electron microscopy (TEM), X-ray diffraction, energy dispersive X-ray spectroscopy (EDX) and Raman Spectroscopy.

It is well-known [29], [30] that the structure of titanate nanoobjects depends on the temperature of the heat treatmen. In this contribution, we demonstrated that the presence of bismuth, antimony and bismuth-antimony can also have an effect of the structural changes. In the case of antimony/titanate sample the recrystallization occurred at higher temperature than in the case of bismuth and bismuth-antimony containing samples.

A combined X-ray diffraction and Raman study confirmed that the pristine and the ion exchanged samples preserved their structure until $500{ }^{\circ} \mathrm{C}$, whereas at higher temperatures they started to collapse and recrystallize into rutile phase via anatase phase. We have shown that the applied metal ions have a strong influence on this recrystallization process; the samples started to collapse and change their crystalline structure at different temperatures depending on the metal content. The presence of bismuth in the titanate sample enhances structural collapse and recrystallization, while antimony acts as a retarding agent in the process. Further investigation is needed to reveal the physico-chemical background of this interesting phenomenon. 


\section{Acknowledgement}

The financial support of the TÁMOP-4.2.2.A-11/1/KONV-2012-0047, TÁMOP-4.2.2.A-11/1/KONV2012-0060 and FP7 INCO „NAPEP” 266600 projects is acknowledged. 


\section{Legend of the Figures:}

Figure 1. TEM image of the as-synthesized titanate nanotubes.

Figure 2. EDX spectra of the pristine (a), antimony (b), bismuth (c), and antimony/bismuth (d) modified titanate nanotubes

Figure 3. TEM images of the pristine (a), antimony (b), bismuth (c), and antimony/bismuth (d) modified titanate nanotubes calcined at $600^{\circ} \mathrm{C}$.

Figure 4. X-Ray diffraction profiles of the pristine (a), antimony (b), bismuth (c), and antimony/bismuth (d) modified titanate nanotube samples. The "*" symbolized the reflection of the sample holder, the " $"$ is the $\mathrm{Sb}_{2} \mathrm{O}_{3}$, the " $\nabla$ " is the $\mathrm{Bi}_{2} \mathrm{O}_{3}$ and the "•" is the $\mathrm{SbBiO}_{4}$.

Figure 5. Raman spectra of the pristine (a), antimony (b), bismuth (c), and antimony/bismuth (d) modified titanate nanotubes. 


\section{References}

[1] R. Cai, J. Chen, J. Zhu, C. Xu, W, Zhang, C. Zhang, W. Shi, H. Tan, D. Yang, H. H. Hng, T. M. Lim and Qingyu Yan, J. Phys. Chem. C, 116 (2012) 12468.

[2] M. E. Spahr, P. S. Bitterli, R. Nesper O. Haas and P. Novák, Journal of The Electrochemical Society, 146 (1999) 2780.

[3] J. H. Park, S. G. Oha and B. W. Jo, Materials Chemistry and Physics, 87 (2004) 301.

[4] N-H. Lee, H-J. Oh, S-C. Jung, W-J. Lee, D-H. Kim and S-J. Kim, International Journal of Photoenergy, 1155 (2011) 327821.

[5] M. Inagaki, N. Kondo, R. Nonaka, E. Ito, M. Toyoda, K. Sogabe and T. Tsumura, Journal of Hazardous Materials, 161 (2009) 1514.

[6] M. Milanović, I. Stijepović and L. M. Nikolić, Processing and Application of Ceramics, 4 (2010) 69.

[7] X. Chang, Z. Liu, C. Li, G. Yang and L. Jia, Nanoscience, 12 (2007) 25.

[8] R. A. Doong and L-F. Chiang, Water Sci., 59 (2008) 1958.

[9] J. Kim and J. Cho, Journal of The Electrochemical Society, 154 (2007) 542.

[10] T. Hirosima and M. Masahiro Angew. Chem. Int. Ed., 44 (2005) 1974.

[11]T. Kasuga, M. Hiramatsu, A. Hosun, T. Sekino and K. Niihara, Langmuir, 14 (1998) 3160.

[12] V. Idakiev, Z.Y. Yuan, T. Tabakova, B.L. Su, Appl. Catal. A, 281 (2005) 149.

[13 ] Z.L. Liu, B. Guo, L. Hong and H.X. Jiang, J. Photochem. Photobiol.

A, 172 (2005) 81.

[14] L. Torrente-Murciano, A. A. Lapkina, D. V. Bavykin, F. C. Walsh and

K. Wilson, Journal of Catalysis, 245 (2007) 272.

[15] Ch. Barglik-Chory, Ch. Remenyi, H. Strohm and G. Müller, J. Phys. Chem. B., 108 (2004) 7637.

[16] J. Cao, J.Z. Sun, H.Y. Li, J. Hong and M. Wang, J. Mater. Chem., 14 (2004) 1203.

[17] X.C. Song, L.H. Yue, B. Liu, G. Han, W.X. Chen and Z.D. Xu, Chinese J. Inorg. Chem., 19 (2003) 899.

[18] H. Tokudome and M. Miyauchi, Chem. Lett., 33 (2004) 1108.

[19] E. Horváth, Á. Kukovecz, Z. Kónya and I. Kiricsi, Chem. Mater., 19 (2007) 927.

[20] Á. Kukovecz, M. Hodos, E. Horváth, G. Radnóczi, Z. Kónya and I. Kiricsi, J.

Phys. Chem. B., 109 (2005) 17781.

[21] M-C. Wu, A. Sápi, A. Avila, M. Szabó, J. Hiltunen, G. Tóth, Á. Kukovecz, Z.

Kónya, R. Keiski, W-F. Su, H. Jantunen and K. Kordás, Nano Research, 4 (2011) 360.

[22] M-C. Wu, J. Hiltunen, A. Sápi, A. Avila, W. Larsson,H-C. Liao, M. Huuhtanen, G. Tóth, A. Shchukarev, N. Laufer, Á. Kukovecz, Z. Kónya, J-P. Mikkola, R. Keiski, WF. Chen, H. Jantunen,P. M. Ajayan, R. Vajtai and K. Kordas, ACS NANO, 5 (2010) 5025.

[23] M-C.Wu,G. Tóth, A. Sápi, A. R. Leino,Z. Kónya, Á. Kukovecz, W. F. Su and K. Kordás, J. Nanosci. Nanotech., 12 (2012) 1421.

[24 ] M. Tóth, J. Kiss, A. Oszkó, G. Pótári, B. László and A. Erdőhelyi, Topics in Catal. 2012, DOI: 10.1007/s 11244-012-9867-7

[25] M. Hodos, E. Horváth, H. Haspel, Á. Kukovecz, Z. Kónya and I. Kiricsi, Chem. Phys. Letters 399 (2004) 512. 
[26] Y.V. Kolenko, K.A. Kovnir, A. I. Gavrilov, A. V. Garshev, J. Frantti, O. I. Lebedev, B.R. Churagulov, G. van Tendeloo and M. Yoshimura, J. Phys. Chem. B 2006, 110, 4030.

[27] X. Sun and Y. Li, Chem-Eur. J., 9 (2003) 2229.

[28] T. Kasuga, M. Hiramatsu, A, Hosun, T.Sekino and K.Nihara, Adv. Mater, 11 (1999) 1307.

[29] G. N. Zhu, C. X. Wang and Y. Y. Xia, Journal of Power Sources, 196 (2011) 2848.

[30] J. Yu, H. Yu, B. Cheng and C. Trapalis, Journal of Molecular Catalysis A: Chemical, 249 (2006) 135. 